\title{
Modeling of Resistive Type Superconducting Fault Current Limiter
}

\author{
Sankalpa Bohidar, Renu Sharma
}

\begin{abstract}
In the coming smart grid, among the important issues related to the application of the "Superconducting Fault Current Limiters" (SFCL) is linked to its potential outcome on the decrease of abnormal fault Current. Because of the increased rate of failure currents, SFCL is most likely to enter a media and low-voltage network to reduce the electrical systems' ability and enhance the strength of the devices. All substations providing the distribution of electrical energy are prone to unnecessary voltage or current spikes, affecting facilities such as interruptions in energy supply, resulting in economic loss for the companies that operate them. Currently, no SFCL Simulink model is implemented in the MATLAB database software. A Matlab / Simulink design for the resistive SFCL is suggested in this research paper. This article first presents a resistive style SFCL model using Matlab / Simulink and then replicates distinct fault kinds and analyzes are performed without SFCL and SFCL. The research demonstrates that SFCL reduces the amount of the fault current to a satisfying stage but also reduces the thermal temporary restoration voltage. SFCL also increases the temporary strength, energy performance and efficiency of energy scheme by spontaneously decreasing error flow. The research paper introduces an advanced current limiter implementation based on superconductivity. The theme of the paper revolves around the design of "resistive-type superconducting fault current limiters".
\end{abstract}

Keywords-substation, distribution system, surges, superconductor.

\section{INTRODUCTION}

The energy consumption in the globe is rising at a large pace, along with India, and the electricity consumption is higher than the production of electricity owing to larger buildings, population growth, water conditioners, larger TVs and more machines. Due to the persistent rise in standard and distributed grid (DG) energy production, such as "solar power plants, photovoltaic crops and wind farms"[1], the probability of an event of failure capable of causing significant and irreparable harm to a large number of electrical equipment, or even a device blackout, has increased quickly. This issue is now a significant concern for the "Transmission System Operators" (TSO), as the enhanced present fault size demonstrates adverse impacts on the safety and efficiency of the entire energy device.

In the upcoming generation, the energy sector is going to be suffered from major technical fluctuations in the medium to the long term agreed in the expert literature. The significant growth of renewable energy, distributed

\footnotetext{
Revised Manuscript Received on 14 August, 2019.

SankalpaBohidar, Department of Electrical Engineering,Institute of Technical Education and Research,Siksha 'O' Anusandhan(Deemed to be University),Bhubaneswar, Odisha, India.

Renu Sharma, Department of Electrical Engineering, Institute of Technical Education and Research, Siksha 'O' Anusandhan(Deemed to be
} University), Bhubaneswar, Odisha, India.(Email: renusharma@soa.ac.in) generation, and smart grids have resulted in a small power system with an independent operation function.

Failure are caused by equipment failure, bad weather, accidents, or intentional vandalism. These types of failures can destroy major and costly components and can lead to long and expensive outages if not erased quickly. One of the greatest auspicious technologies in this application is a superconducting material with a high critical temperature (i.e. liquid nitrogen temperature> 77K). Superconducting fault current limiter is dependent on the basic properties of a superconductor [2]-[6]. With the exceeding of the threshold value of current of the material, the property also changes from super-conducting state to normally operating state.

If a failure occurs in one of these weaknesses, a power interruption can enter the network. The system is designed to avoid these interruptions. The purpose is to provide electrical energy with little or virtually no resistance (a property known as superconductivity) and virtually zero for losses in electrical energy. A system that represents a trendy study of superconducting technology [7] used to develops focusing on the initiative of the "European project ECCOFLOW" [8] and implemented in electrical networks. Its main purpose is to design current limiter circuit based on the second-generation high temperature (HTS) [9] cable and is named SFCL.

The SFCL concept focuses on superconductivity. It claims that - "any superconducting material is in the superconducting stage as long as the temperature, current and magnetic field density are below its critical limit". Additionally, whether critical values that define swapping among the superconducting state to the normal condition, such as "critical current density (Jc), critical temperature (Tc), or critical magnetic field (Hc)", are exceeded, then SFCL resistance is added to the circuit to eliminate the fault current magnitude.

The continuously increasing demand for electrical energy had led to an increase in the size of generating stations along with distribution network which is also known as power grids. The increase further leads to uncharacteristic operation. The traditional circuit breakers are not applicable in the network because the upraise in fault current levels may reach its breaking capacity limit. This upraises results in the replacement of a huge number of devices used in power systems such as circuit breaks and transformers. One of the innovative alternatives in order to prevent the issue of rising fault current is super-conducting Fault Current Limiter (SFCL). It increases the efficiency and safety of the transmission system by automatically increasing the fault

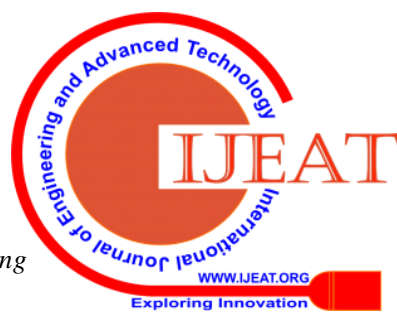


present. SFCLs provide good impedance and very small impedance under ordinary situations, and fast restoration to null impedance after clearance of malfunction.

The latest fault limit (SFCL) has different features derived from superconductors ' structures. This paper discusses fundamental aspects of superconductivity utilized to demonstrate how the electrical resistance belonging to the high-temperature supreme conductors arises in the fluxflow system. Super conductance is a situation defined by a strong contact among conduit electrons. This strong association decreases the entropy of the process and facilitates the physical movement of coupled electrons at considerable lengths in this particular state which exists in other elements of the periodical framework.

This long-term coherence period is deemed accountable for the optimal conductance of superconductors. In contrast to the "zero resistance brand", the complete and reversible "diamagnetism" characterizes the ideal superconductors. The "Meissner effect, i.e. the lack of magnetic flux in the product bulk in any preliminary circumstances is the unique action". The "Meissner effect" is the "expulsion of the magnetic field from a superconductor during its transition to the superconducting state".

Nonetheless, with the capacity of superconductors to raise their impedance easily, a "superconducting fault current limiter" could solve such limitations, which operate just marginally or not up until a fault current event takes place.

Thus, consumption is increasing at an extremely high level and power production runs ahead of availability. The key shift to the "distribution network" is the implementation of "decentralized energy resources" (DES). DES has been gradually combined with the "distribution network "utilizing adapters to satisfy the ever-greater need for electricity.

The adoption rates of decentralized energy supplies are projected to drastically upsurge in future. As decentralized energy resources are linked to the "distribution network", the probability of breakdown current decreases, which can contribute to inconsistencies in the whole electricity network. Equipment being installed newly at the power grid or sub-station is very costly to maintain. Hence it is essential to protect devices from the fault current.

This research paper is categorized into several chapters, wherein chapter II tells about modeling of SFCL, chapter III discusses the experiments and their respective results. The end chapter concludes all the work done in the paper.

\section{LITERATURE REVIEW}

The major field of this research was to sever the loop through fault before research and development of fault current restricting system so that the costly apparatus on power grids could be safeguarded from high fault currents. The "circuit breaker" with a high rating was designed to withstand strong fault currents. But, the trouble with the "circuit breakers" seems to be that their existence is short, so they won't break the loop before the initial current phase is zero. "Fault current limiters" were studied and established several years ago. The Traditional "Superconducting Fault Limiter (SFCL") has special attributes found in the nature of strongly non-linear superconductors.

The study of the SFCL principles and the multiple SFCL forms and to some degree the fault present limiting variable was clarified and contrasted to SFCL's by [10]. The "Superconducting Fault Current Limiters" (SFCLs) increases the device trust and flexibility by automatically reducing the fault current. Papers [11], [12]demonstrates the entire system performance by modifying the present trigger rate along with a synchronous generator in such an artificial transmission cable. It states the SFCLs boost transient stabilization. Paper [13] suggested a "non-inductive HTS wire coil" that was both resistive and inductive throughout the parallel manufacturing processes. Impedance properties are shown in the "short circuit" test performance.

Paper [14]describes the various types and attributes of SFCLs. Specific features and drawbacks of the existing "resistive superconducting" defects are addressed. In multiple testing conditions, paper [15]displays the observational quench effects of "YBCO Coated Conductors" for average distances. The findings suggest that "YBCO CCs" wire was feasible for resistive SFCL applications. The optimum discovery of "CC" for "FCL" is presented by paper by the two various types of "YBCO CCs"[16]-[18]. The experimental findings were correlated with the standard restricting properties of "CC" leveraging the "Finite Element Method" (FEM) for short circuit experiments in these specimens. Paper [19], [20] suggested a "2MVA" medium voltage applications fault current restricting unit of " 750 " meters of "AMSC's 344S" supremacy conductors, the embossed " 2 G HTS" stainless steel wire. The current restricting features of the "2 G HTS" wire are demonstrated by energy measurements and dielectric examinations. Paper [21] performed tests at SuperPower with " $138 \mathrm{kV}$ ", "2 G SFCL". The paper describes the experiments and modifications to "maximize load recovery" (RUL) for the concept of the viability of RUL. This makes the choice of reduced superconductor material safer, which decreases volume and total conduction damages. Regarding purposes of the thermal or electric-magnetic behavior, paper [9] suggests a modern finite component framework for the integrating the electrical and thermal equations throughout the "single solver for a high-temperature superconductor". The coated conductor is a structural conductor that influences the existing restricting attributes of surface materials, HTS, ground, etc. Papers [22]-[24]illustrates a feasible HTS as an SFCL for the analysis of commercially available CCs. Paper [25], [26] conducted an SFCL design trial of " $10 \mathrm{kV} / 200 \mathrm{~A}$ " and carried out several experiments involving short circuit testing, recovery tests and "LN2" boiling trials. The findings indicate that the fault current gets decreased by " $30 \%$ " to " $70 \%$ ", while the time to return from the fault span was linear. 


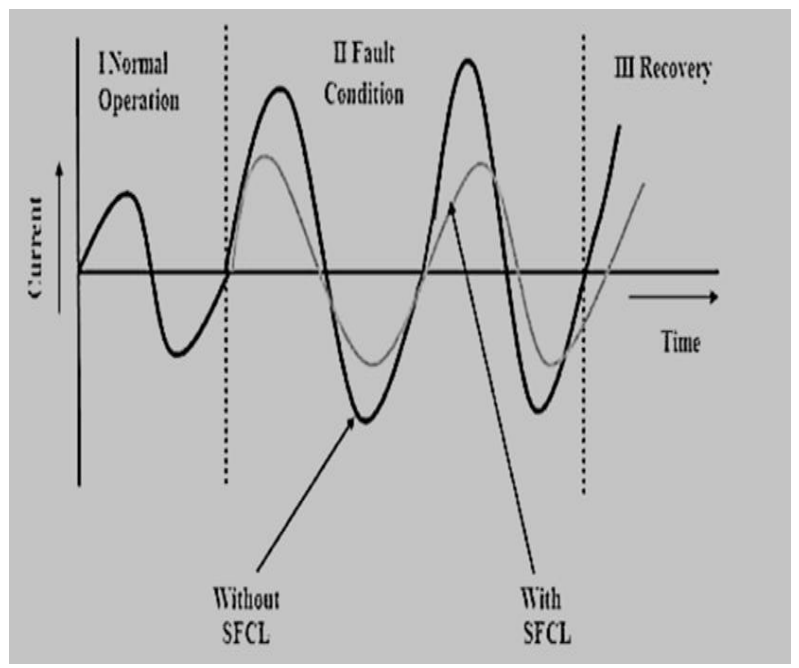

Figure 1 showcases the current during a normal situation and in fault situations.

As per a "Smart Grids report from Morgan Stanley", the potential fault-sourcing technology market that hit "USD 5 billion" per annum by 2030, but further research is still required on the specific effects of SFCL deployment on power systems. But further analysis of the actual impact on electricity networks of installing SFCLs remains needed. Throughout the previous work [11 ], showing the feasibility/ practical approach of this technique through a rigorous analysis of the effective field test and the numerous current SFCL mathematical models.

Two generalized SFCL systems have often been used for output modeling of SFCLs deployed in actual grids. The first solution was to construct the SFCL as the phase resistance, as in the reference [27], [28] with the pre-defined triggering present, timing and regeneration period. This method helps one of them to take note of a simpler situation where there will be no energy leakage throughout the superconducting phase and when the SFCL reacts to defects automatically, large impedance is seen in its regular state simulation.

And from the other side, it is also possible to simplify the simulation of SFCL in power grid by utilizing an "exponential function for an SFCL system" dynamic force, where the quenching effect of the superconducting material is primarily defined by time. This approach was already used in Refs.[29]to determine the best position for a power grid and related cost for a wind turbine generation system SFCLs found that SFCLs can minimize, however can also intensely increase, the efficiency of wind farm SFCLs can also be significantly improved.

This incremental resistance curve best adapts to the actual output of SFCL and gives robust statistical advantages regarding quantitative consistency in contrast with the "Heaviside phase method" developed from the previous strategy. However, until the simulation is begun, the SFCL parameters, including that of the "firing, quenching and recovery period, must also be set", i.e., the physical attributes of superstructures are neglected in this scenario as well.

\section{MODELING OF SFCL (SUPERCONDUCTING FAULT CURRENT LIMITER)}

The main aim serves to fit this system into the distribution network substation. The study on this proposed "superconducting fault current" under actual distribution situations is novel. The goal of SFCL installation is improving the operation, quality of supply, efficiency, and stability of the electrical network without the need to build additional substations or new infrastructure. The main objective helps in ensuring that the system has been tuned for optimal operation, to verify operation and to analyze the results over the medium to long term. This paper helps in analyzing the performance of Spanish distribution substations under conventional operating conditions[30], [31].

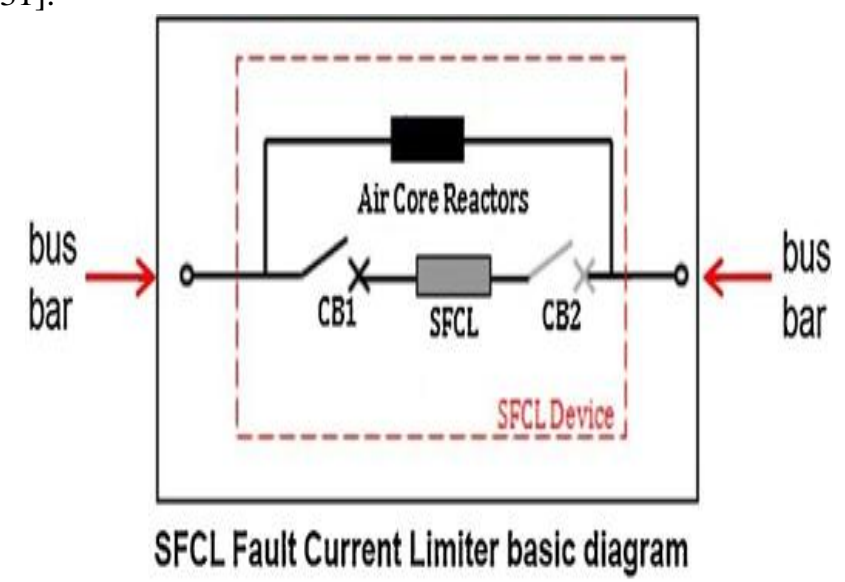

This design is being optimized for subsequent highvolume production of superconducting modules and requires specific coolant temperatures depending on the material used as the superconductor [32]. The temperature lies within the range of $65 \mathrm{k}$ and $80 \mathrm{k}$ for copper compound material. At this temperature, the material acquires superconducting properties. In contrast, for other materials such as $\mathrm{MgB}_{2}$, the temperature range should be $20 \mathrm{~K}-30 \mathrm{~K}$. The material selected for this project is YBCO composite material [33], [34] material properties, scientifically represented as SF12100. liquid nitrogen is used as refrigerant [35]. This is because to meet one of the required specifications and is simpler and cheaper solutions compared to other refrigerants. Its main advantage is the ease of marketing and acquisition in the market.

With the designing of the system, the number of properties comprising a superconducting material:

- It can withstand high-strength short circuits to superconducting materials and recovers its initial state after a certain period of time after the overload stops.

- $\quad$ There must be a negligible loss in current due to small distance between the bands that makes up the module.

- $\quad$ For greater than nominal values of voltages there must be a tried and test.

- The thermal interface is designed and optimized for most agents that are used as refrigerants, reducing recovery time. 


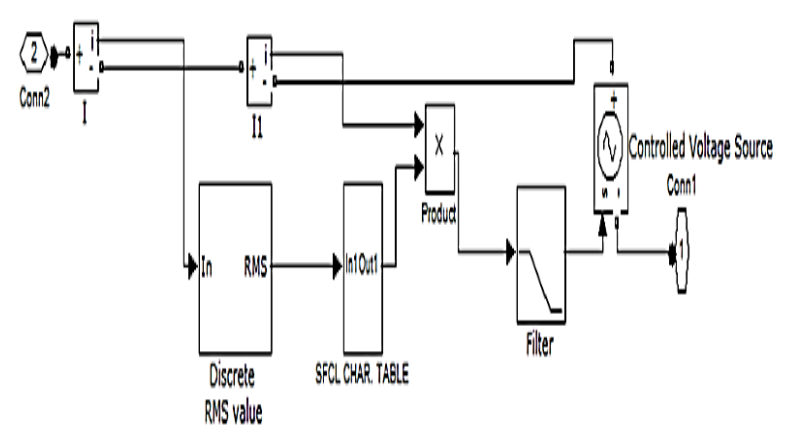

Fig.1(a): SFCL Simulink Model

Figure 1 (a) shows a SFCL model developed with the Simulink / Sim Power system. Thefunctions of the SFCL model are as follows. Firstly, the RMS block calculates the magnitude of flowing current, and further, the calculated value is compared with the characteristic of SFCLtable. Figure 2 (b) shows the SFCL characteristic table subsystem. If current flowing exceeds the trigger current level, the SFCL resistance will increase to a level with maximum impedance within a threshold time called response time.

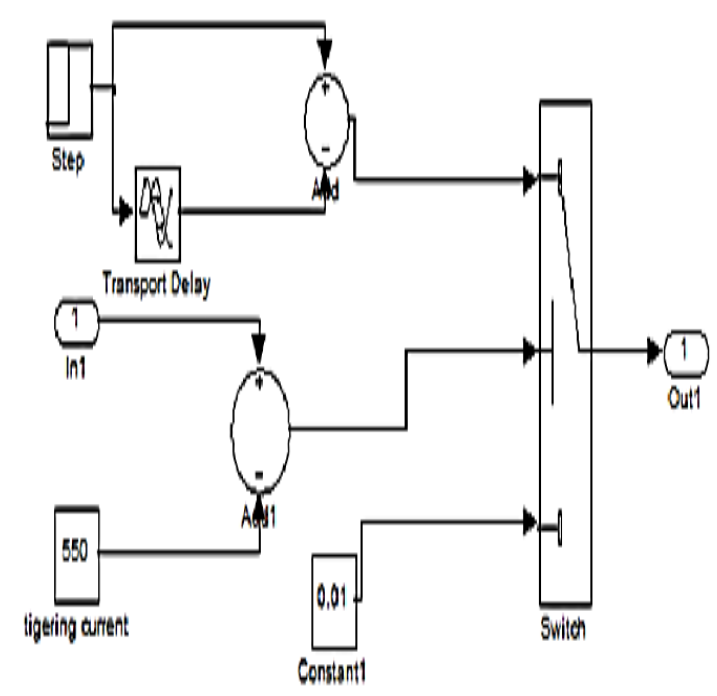

Fig.1(b): SFCL Characteristic Table

The increase in the value of impedance leads to decreasing the short-circuit fault current. However, the SFCL resistance is again minimized when current is below trigger current level.

\section{RESULTS AND DISCUSSIONS}

\section{A. Without SFCL}

Figure 2(a) shows current flowing through circuit breaker contacts before the current is zero when FCL is not applied. As apparent from Figure 2(b), the highest value of fault current is calculated as $3500 \mathrm{~A}$, which can eventually damage the system. TRV without FCL reaches a maximum value of $24 \mathrm{kV}$. The "initial transient recovery voltage" (ITRV) without FCL reaches the first peak of $23.7 \mathrm{kV}$ in 0.0044 seconds. From these values, RRRV is $55 \mathrm{kv} / \mu \mathrm{s}$.

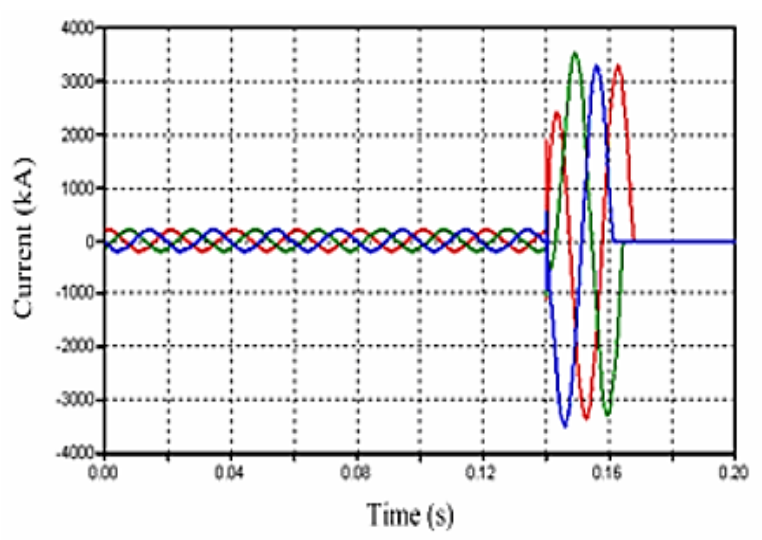

Fig.2(a) Circuit Breaker Without FCL

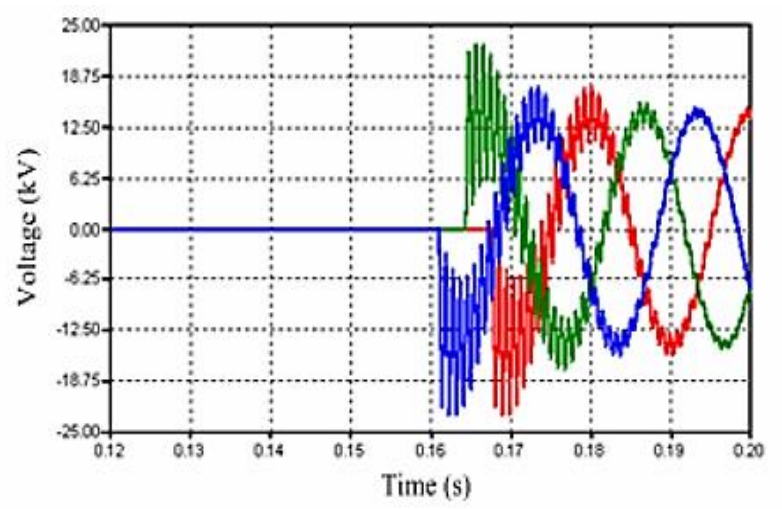

Fig.2(b)TRV Circuit Breaker Without FCL

\section{B. $\quad$ Using Resistive-SFCL}

Figure 3 (a) shows current through circuit breaker contacts before the current is zero in a resistor SFCL application. As seen from Figure 3 (b), the highest value of the fault current gets limited to approximately 650A. Application of resistive-SFCL aims in eliminating the asymmetric component of fault current. Figure 7 (f) shows the TRV of circuit breaker onto which resistive-SFCL can be applied. TRV reaches maximum value of $16 \mathrm{kV}$. The "initial transient recovery voltage (ITRV)" using inductiveSFCL reaches the first maximum value of $7 \mathrm{kV}$ in 0.0009 seconds. From these values, RRRV was reduced to $8 \mathrm{kv} / \mu \mathrm{s}$.

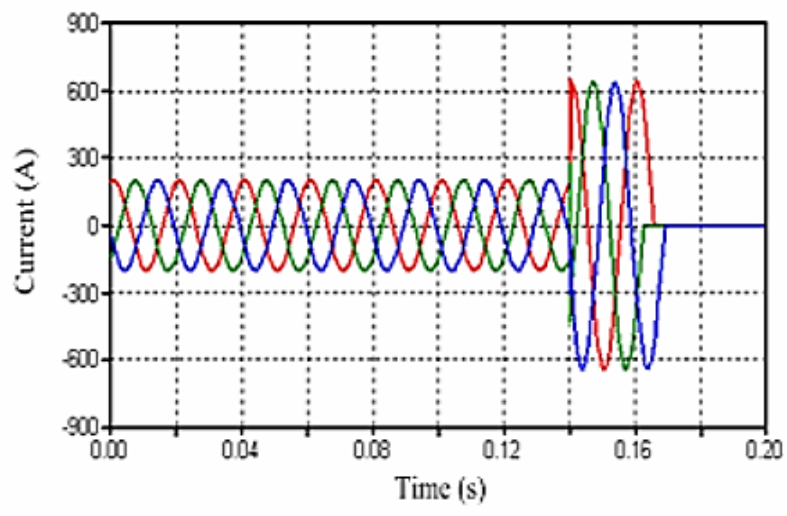

Fig.3(a) Current Circuit Breaker Using Resistive SF

Published By: Blue Eyes Intelligence Engineering 


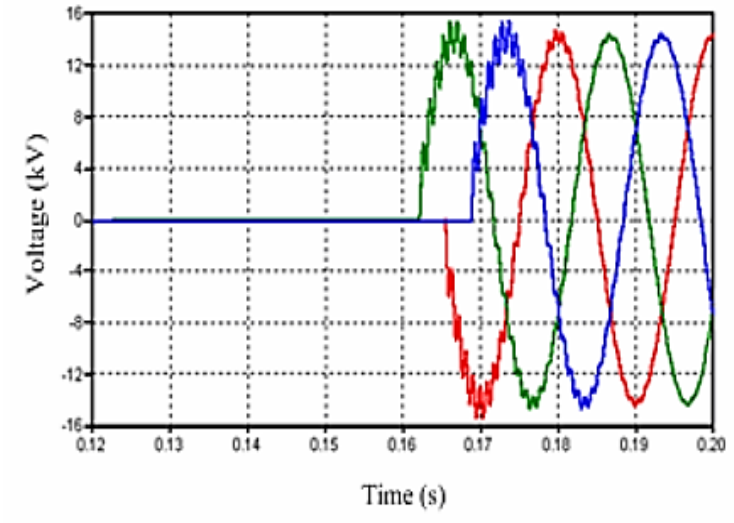

Fig.3(b) TRV Circuit Breaker Using Resistive SFCL

\section{CONCLUSION}

SFCL is recognized successful tool to reduce gain in error levels induced by developing the power system and the inclusion of sustainable energy technologies. In this research paper, the SFCL model is incorporated into a straightforward three-phase scheme composed of origin, charge and measuring frames. The modeled present waveforms of this embedded system were evaluated under distinct working circumstances. The outcomes are tabulated in a chart that demonstrates that the temporary increase in fault current utilization the SFCL module is reversed to the required price utilizing SFCL.SFCL prototype and simulation models were done in MATLAB. This model can be tested experimentally for novel and efficient design of SFCL application in the present electrical networks. In the paper, the proposed system is developed in which results obtained with and without realizing "resistive type superconducting fault current limiter" with a current circuit breaker is compared successfully.

\section{REFERENCES}

1. V. Siva Reddy, S. C. Kaushik, K. R. Ranjan, and S. K. Tyagi, "State-of-the-art of solar thermal power plants - A review," Renewable and Sustainable Energy Reviews. 2013.

2. S. S. Kalsi, "Power Applications: Fault Current Limiters," in Applied Superconductivity: Handbook on Devices and Applications, vol. 2-2, 2015, pp. 631-644.

3. W. T. B. De Sousa, A. Polasek, R. Dias, and R. de Andrade, "Superconducting fault current limiters: Main concepts and tests," Rev. Bras. Ensino Fis., vol. 34, no. 4, 2012.

4. A. Etxegarai, I. Zamora, G. Buigues, V. Valverde, E. Torres, and P. Eguia, "Models for Fault Current Limiters based on Superconductor Materials," Renew. Energy Power Qual. J., pp. 284-289, 2016.

5. V. Meerovich and V. Sokolovsky, "High-temperature superconducting fault current limiters (FCLs) for power grid applications," in Superconductors in the Power Grid: Materials and Applications, 2015, pp. 283-324.

6. S. Patil and A. Thorat, "Development of fault current limiters: A review," in 2017 International Conference on Data Management, Analytics and Innovation, ICDMAI 2017, 2017, pp. 122-126.

7. M. C. Ahn, D. K. Park, S. E. Yang, and T. K. Ko, "Impedance characteristics of non-inductive coil wound Transactions on Applied Superconductivity, 2008. with two kinds of HTS wire in parallel," in IEEE

8. K. Yamabe, N. Yonemura, Y. Shirai, and J. Baba, "Current limiting and recovery tests Under Load of threephase transformer type coaxial SFCL in a model power system," IEEE Trans. Appl. Supercond., 2014.

9. S. V. Muravyov, V. N. Borikov, and N. M. Natalinova, "A computer measurement of welding surge currents," Meas. Control, vol. 42, no. 2, pp. 44-47, 2009.

10. V. Dabeer, "Fault current limiters," Water and Energy International. 2016.

11. S. Rüberg, A. L'Abbate, G. Fulli, and A. Purvins, "HighVoltage Direct-Current Transmission," Power Syst., 2013.

12. D. Elliott et al., "A Comparison of AC and HVDC Options for the Connection of Offshore Wind Generation in Great Britain," IEEE Trans. Power Deliv., 2016.

13. H. W. Kim et al., "Enhancement of 2G HTS coil stability with V2O3 and perforated HTS wire," IEEE Trans. Appl. Supercond., vol. 28, no. 3, 2018.

14. Q. Yang, S. Le Blond, F. Liang, W. Yuan, M. Zhang, and J. Li, "Design and Application of Superconducting Fault Current Limiter in a Multiterminal HVDC System," IEEE Trans. Appl. Supercond., 2017.

15. K. Matsumoto and P. Mele, "Artificial pinning center technology to enhance vortex pinning in YBCO coated conductors," Supercond. Sci. Technol., 2010.

16. L. Jaroszyński and T. Janowski, "YBCO coated conductors for superconducting transformer windings," Prz. Elektrotechniczny, vol. 90, no. 1, pp. 164-166, 2014.

17. L. Jaroszyński and D. Czerwiński, "Numerical analysis of YBCO coated conductors," Prz. Elektrotechniczny, vol. 88, no. 10 B, pp. 160-162, 2012.

18. J. Lu, K. Han, W. R. Sheppard, Y. L. Viouchkov, K. W. Pickard, and W. D. Markiewicz, "Lap joint resistance of YBCO coated conductors," IEEE Trans. Appl. Supercond., vol. 21, no. 3 PART 3, pp. 3009-3012, 2011.

19. T. Feehally et al., "Battery energy storage systems for the electricity grid: UK research facilities," in IET Conference Publications, 2016.

20. C. Concari, P. Cova, G. Franceschini, A. Toscani, and F. Bertoluzza, "Improved control strategy for modular 2MVA AC/AC power converter," in 2013 IEEE Energy Conversion Congress and Exposition, ECCE 2013, 2013.

21. A. Morandi et al., "Design of a DC resistive SFCL for application to the $20 \mathrm{kV}$ distribution system," IEEE Trans. Appl. Supercond., 2010.

22. W. T. B. De Sousa, T. M. L. Assis, A. Polasek, A. M. Monteiro, and R. De Andrade, "Simulation of a superconducting fault current limiter: A case study in the Brazilian power system with possible recovery under load," IEEE Trans. Appl. Supercond., vol. 26, no. 2, 2016.

23. H. P. Kraemer et al., "Superconducting fault current limiter for transmission voltage," in Physics Procedia, 2012, vol. 36, pp. 921-926.

24. M. Majka and J. Kozak, "Superconducting fault current limiter for the electric power system," in Acta Physica Polonica A, 2016, vol. 130, no. 2, pp. 581-584.

25. L. Chen, Y. J. Tang, M. Song, J. Shi, and L. Ren, "Parameter design and performance simulation of a 10 $\mathrm{kV}$ voltage compensation type active superconducting fault current limiter," Phys. C Supercond. its Appl., 2013.

26. Y. Teng et al., "Analysis on heat loss characteristics of a $10 \mathrm{kV}$ HTS power substation," Cryogenics (Guildf)., 2014.

27. [27] Z. C. Zou, X. Y. Chen, C. S. Li, X. Y. Xiao, and Y. Zhang, "Conceptual Design and Evaluation of a Resistive-Type SFCL for Efficient Fault Ride Through in 
a DFIG," IEEE Trans. Appl. Supercond., 2016.

28. G. Didier and J. Lévêque, "Influence of fault type on the optimal location of superconducting fault current limiter in electrical power grid," Int. J. Electr. Power Energy Syst., 2014.

29. X. Zhang et al., "Power flow analysis and optimal locations of resistive type superconducting fault current limiters," Springerplus, 2016.

30. S. Dutta and B. C. Babu, "Modelling and analysis of resistive Superconducting Fault Current Limiter," in IEEE TechSym 2014 - 2014 IEEE Students' Technology Symposium, 2014.

31. W. Schmidt, H. P. Kraemer, H. W. Neumueller, U. Schoop, D. Verebelyi, and A. P. Malozemoff, "Investigation of YBCO coated conductors for fault current limiter applications," in IEEE Transactions on Applied Superconductivity, 2007.

32. S. Nemdili and S. Belkhiat, "Electrothermal modeling of coated conductor for a resistive superconducting faultcurrent limiter," J. Supercond. Nov. Magn., 2013.

33. S. Kar, S. Kulkarni, S. K. Sarangi, and V. V. Rao, "Conceptual design of a $440 \mathrm{~V} / 800$ a resistive-type superconducting fault current limiter based on high T c coated conductors," IEEE Trans. Appl. Supercond., 2012.

34. S. Kar, S. Kulkarni, S. K. Sarangi, and V. V Rao, "Conceptual Design of a 440 V/800 A Resistive-Type Superconducting Fault Current Limiter Based on High $\langle$ formula formulatype="inline" $\rangle\langle$ img src="/images/tex/801.gif" alt="T_\{c $\} "></$ formula $>$ Coated Conductors," Appl. Supercond. IEEE Trans., 2012.

35. K. Nam, H. Kang, C. Lee, T. K. Ko, and B. Y. Seok, "Visualization study on boiling of nitrogen during quench for fault current limiter applications," in IEEE Transactions on Applied Superconductivity, 2006. 